ARTICLE

Received 12 Mar 2015 | Accepted 27 May 2015 | Published 6 Jul 2015

DOI: $10.1038 /$ ncomms8641 OPEN

\title{
The circadian clock rephases during lateral root organ initiation in Arabidopsis thaliana
}

Ute Voß ${ }^{1, \star}$, Michael H. Wilson ${ }^{1, \star}$, Kim Kenobi $^{1, \star}$, Peter D. Gould ${ }^{2}$, Fiona C. Robertson ${ }^{3, \dagger}$, Wendy A. Peer ${ }^{4}$, Mikaël Lucas ${ }^{1,5}$, Kamal Swarup¹, Ilda Casimiro ${ }^{6}$, Tara J. Holman', Darren M. Wells ${ }^{1}$, Benjamin Péret ${ }^{1,7}$, Tatsuaki Goh ${ }^{1,8}$, Hidehiro Fukaki ${ }^{8}$, T. Charlie Hodgman ${ }^{1}$, Laurent Laplaze ${ }^{5}$, Karen J. Halliday 9,10 , Karin Ljung ${ }^{11}$, Angus S. Murphy ${ }^{12}$, Anthony J. Hall ${ }^{2}$, Alex A.R. Webb ${ }^{3} \&$ Malcolm J. Bennett ${ }^{1}$

The endogenous circadian clock enables organisms to adapt their growth and development to environmental changes. Here we describe how the circadian clock is employed to coordinate responses to the key signal auxin during lateral root (LR) emergence. In the model plant, Arabidopsis thaliana, LRs originate from a group of stem cells deep within the root, necessitating that new organs emerge through overlying root tissues. We report that the circadian clock is rephased during LR development. Metabolite and transcript profiling revealed that the circadian clock controls the levels of auxin and auxin-related genes including the auxin response repressor IAA14 and auxin oxidase AtDAO2. Plants lacking or overexpressing core clock components exhibit LR emergence defects. We conclude that the circadian clock acts to gate auxin signalling during LR development to facilitate organ emergence.

\footnotetext{
${ }^{1}$ Centre for Plant Integrative Biology, School of Biosciences, University of Nottingham, Sutton Bonington Campus, Sutton Bonington, Nottingham LE12 5RD, UK. ${ }^{2}$ Department of Comparative and Functional Genomics, Institute of Integrative Biology, University of Liverpool, Liverpool L69 7ZB, UK. ${ }^{3}$ Department of Plant Sciences, University of Cambridge, Cambridge CB2 3EA, UK. ${ }^{4}$ Department of Environmental Science \& Technology, College of Agriculture \& Natural Resources, University of Maryland, College Park, Maryland 20742, USA. ${ }^{5}$ Institut de Recherche pour le Développement, UMR DIADE, Montpellier 34394 , France. ${ }^{6}$ Departamento Anatomía, Biología Celular Y Zoología, Facultad de Ciencias, Universidad de Extremadura, Badajoz 06006, Spain. ${ }^{7}$ UMR 7265 CEA, CNRS, Université d'Aix-Marseille, Laboratoire de Biologie du Développement des Plantes, Saint-Paul-lez-Durance 13108, France. ${ }^{8}$ Department of Biology, Graduate School of Science, Kobe University, Kobe 657-8501, Japan. ${ }^{9}$ School of Biological Sciences, University of Edinburgh, Edinburgh EH9 3JH, UK.

10 SynthSys, CH Waddington Building, University of Edinburgh, Edinburgh EH9 3JD, UK. ${ }^{11}$ Umea Plant Science Centre, Department of Forest Genetics and Plant Physiology, Swedish University of Agricultural Sciences, Umea SE-901 83, Sweden. ${ }^{12}$ Department of Plant Science and Landscape Architecture, University of Maryland, Maryland 20742, USA. * These authors contributed equally to this work. † Present address: Department of Biochemistry, University of Zimbabwe, PO Box MP 45, Mount Pleasant, Harare, Zimbabwe. Correspondence and requests for materials should be addressed to M.J.B.

(email: malcolm.bennett@nottingham.ac.uk).
} 
T he circadian clock plays a key role in controlling plant growth and development. Genetic studies in the model plant Arabidopsis thaliana have revealed that the circadian clock is composed of interacting repressing loops. The core loop consists of the morning loop genes, LATE ELONGATED HYPOCOTYL (LHY) and CIRCADIAN CLOCK-ASSOCIATED 1 (CCA1), which repress the evening loop gene TIMING OF CAB EXPRESSION 1 (TOC1), which in turn represses both morning and evening loop genes ${ }^{1-6}$ (reviewed in ref. 3).

In plants, the circadian clock is traditionally considered to be cell autonomous but there is emerging evidence of synchronization between the oscillators in individual cells ${ }^{7}$. It has been shown that the plant clock is organ-specific but not organ-autonomous, however, the mechanisms by which circadian rhythms become established or how the circadian clock synchronizes between cells currently represent important areas of research.

Lateral roots (LR) provide an ideal system to study how adjacent populations of plant cells synchronize their growth and development (reviewed in ref. 9). In Arabidopsis, LRs are derived from pairs of xylem pole pericycle founder cells located deep within the primary root. These cells undergo several rounds of anticlinal, periclinal and tangential cell divisions to form a new LR primordium ${ }^{10}$. Auxin is a major regulator in this process ${ }^{9}$.To emerge into the soil, a LR primordium first has to break through overlying endodermal, cortical and epidermal cells. This is achieved by employing the hormonal signal auxin to reprogramme overlying cells to undergo cell separation and facilitate the emergence of the new root organ ${ }^{11-13}$.

In this current study, we generated a transcriptomic time course data set of LR development and found that the circadian clock is rephased during LR initiation and that its regulation of auxin-related components acts to control the rate of LR emergence. Disrupting clock function or oscillatory expression of downstream auxin-related targets impairs LR emergence.

\section{Results}

All core circadian clock genes oscillate in LR primordia. To identify novel genes and mechanisms regulating LR development, we initially generated a transcriptomic time course data set covering every stage of LR development. We previously showed that, following a $90^{\circ}$ gravitropic stimulus, LRs develop in a highly synchronized manner at the outer edge of a bending root (Fig. 1a,b $)^{14}$. In Col-0 roots, under our growth conditions, stage I LR primordia are detected at $15 \mathrm{~h}$ post gravity induction (pgi) and LRs emerged $42 \mathrm{~h}$ pgi (Fig. 1a,b). We took advantage of this regular LR primordium development by microdissecting root bends every $3 \mathrm{~h}$ from 6 to $54 \mathrm{~h}$ pgi (Fig. 1b,c). For this, Col-0 seedlings were grown without previous entrainment in constant light conditions for 3 days, before the gravity stimulus was applied. From four independent biological replicates of each time point, Affymetrix transcript abundance data were generated, encompassing every stage from pre-initiation to post-emergence (Fig. 1b; microarray data are publicly available at ArrayExpress (www.ebiac.uk/ arrayexpress/) under the accession number E-MTAB-2565; all data shown in this manuscript are averages from those four replicates). Initial quality control testing confirmed the ability of the data set to identify LR development regulatory genes. We examined the transcript abundance of several known key LR regulators: AUXIN RESPONSE FACTOR (ARF) 19, LATERAL ORGAN BOUNDARIES DOMAIN (LBD) 16, 18 and 29, SHORT HYPOCOTYL 2 (SHY2), GATA TRANSCRIPTION FACTOR 23 (GATA23), CYCLIN B1;1 (CYCB1;1) and LIKE AUX1 3 (LAX3) (Supplementary Fig. 1), which were all significantly upregulated at previously published time points ${ }^{13,15-19}$. In total, $>8,000$ genes were differentially expressed across all time points and principal component $(\mathrm{PC})$ analysis revealed an oscillatory pattern in the third PC with a period of $\sim 24 \mathrm{~h}$ (PC3; Fig. 1d ${ }^{20}$. Clustering of transcript abundance profiles generated a set of 77 clusters, revealing a broad range of gene mRNA patterns during LR a

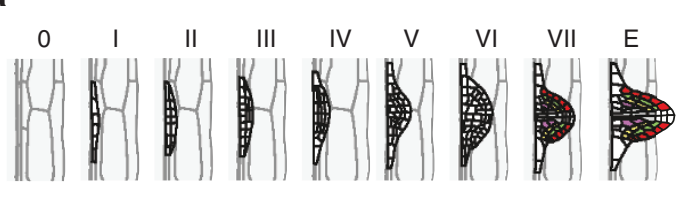

C

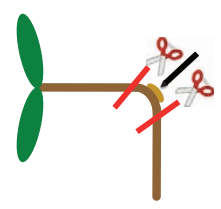

b

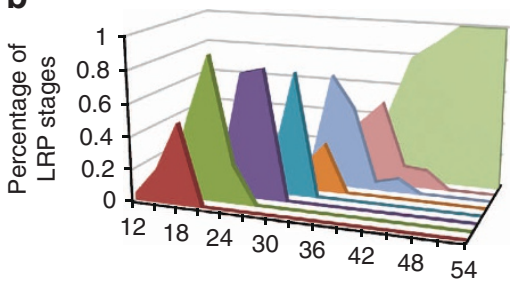

Time after gravitropic stimulus ( $h$ )

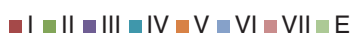
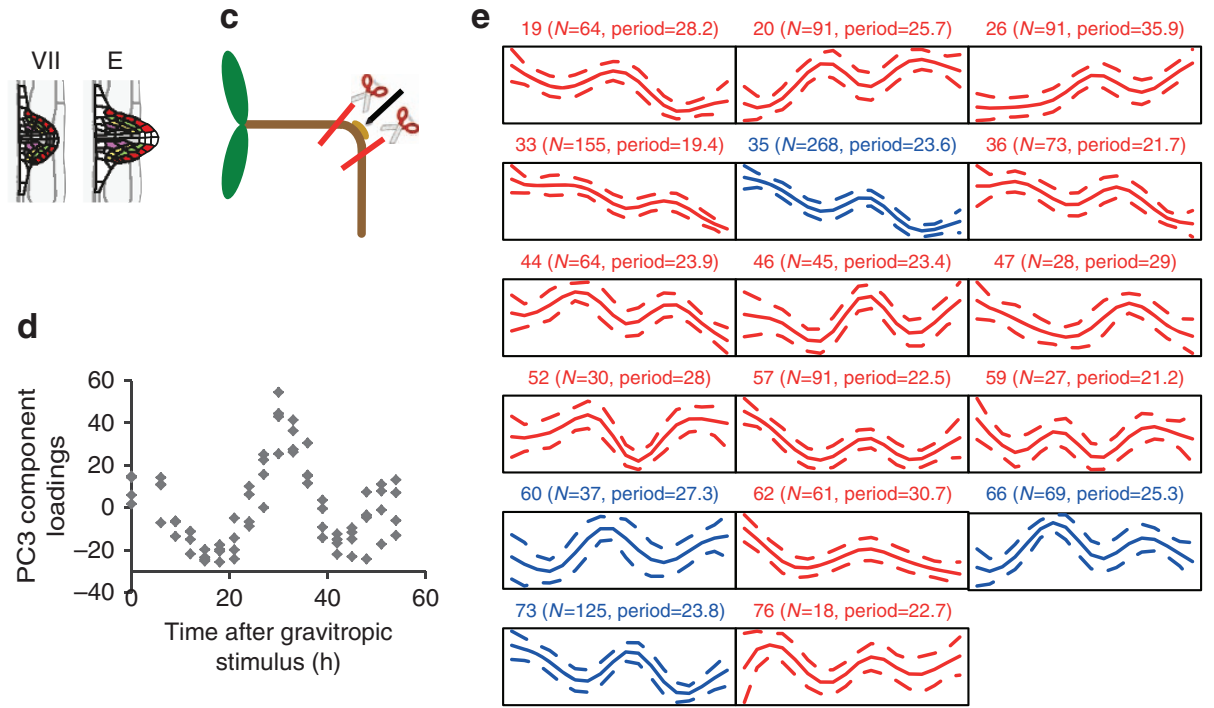

Figure 1 | Generation of a lateral root transcriptomic data set. (a) Overview of lateral root primordium stages, as described in ref. 10. Image adapted from ref. 14. (b) Gravitropic stimulation was used to induce the synchronized initiation of lateral root primordia at the site of root bending in a population of 3-day-old seedlings. Lateral root primordium stages (from I to VIII according to previous descriptions from ref. 10) were determined every $3 \mathrm{~h}$ from 6 to $54 \mathrm{~h}$ post gravity induction and are represented here as a percentage of the total number of observed lateral root primordia at each time point ${ }^{14}$. (c) Root bends were microdissected for each of those 18 time points and used for RNA extraction and subsequent microarray analysis ( $N=450$ per time point, 4 independent biological replicates). All seedlings were grown in constant light conditions, without previous entrainment. Image is adapted from ref. 14. (d) Principal component analysis of the lateral root data set revealed an oscillating component in the third principal component (PC3). (e) When differentially expressed genes were clustered, 17 clusters showed an oscillatory pattern. The estimate of the period length ( $h$ ) and the number of genes in each cluster $(N=x)$ is indicated above each cluster. Clusters with blue lines comprise core circadian clock genes. Expression intensities are on a log2 scale. The mean of the clusters is given by the solid line and the dotted lines show the mean \pm 2 s.d. 
development (Supplementary Fig. 2 and Supplementary Data 1). Consistent with PC3, 17 clusters exhibited an oscillatory pattern with a period ranging from 19.4 to $35.9 \mathrm{~h}$ (Fig. 1d,e; Supplementary Fig. 2), and four oscillating clusters comprised well-characterized core circadian clock genes (blue in Fig. 1e and Supplementary Fig. 2; cluster 35 contains ELF3, cluster 60 GI and PRR7, cluster $66 C C A 1$ and $L H Y$, cluster $73 T O C 1, P R R 5$, $P R R 3$ and ELF4; the individual transcript abundance patterns of these nine core circadian clock genes are shown in Fig. 2).

To validate that the LR transcriptome displays circadian oscillations under free-running conditions, we analysed the data set using the JTK_CYCLE algorithm ${ }^{21}$. Rhythmic components were identified in the mRNA patterns of 1,575 genes, including all the core circadian clock genes, with an average period length of $25.3 \mathrm{~h}$ (Supplementary Data 2). As such a large proportion of genes were oscillating close to a 24 -h period in the data set, we investigated the behaviour of the set of nine core circadian clock component genes in more detail (Fig. 2). All nine genes clearly showed oscillatory transcript abundance, and the period of rhythms in $L H Y$ and CCA1 mRNAs in the LR is similar to the period of their expression measured in whole roots ${ }^{8}$ (Supplementary Data 2). Morning loop genes including CCA1 and LHY were oscillating in anti-phase to the evening loop gene TOC1 (indicated by vertical red lines in Fig. 2), indicating that we are observing a fully operating circadian clock in LR development.

It has been previously described that only the day loop is fully operating in Arabidopsis roots, synchronized by photosynthetic signals derived from the shoot circadian oscillator ${ }^{8}$. The most likely difference with our observations is due to different growth conditions. In the cited work, plants were grown hydroponically in light-tight boxes. Therefore, under constant light conditions, the leaves were in constant light but the roots were in constant darkness. In our experiments, both roots and leaves are exposed to constant light. Another difference is that whole roots were analysed ${ }^{8}$, whereas here we follow the development of LR primordia. When entrained in light-dark cycles the day-loop reporter TOC1:LUC oscillates in primary and lateral roots while in constant dark conditions waves of circadian oscillations travel from the root tip upwards ${ }^{22}$. This principle is very similar to that of a non-circadian oscillation of auxin responsive gene expression, such as $A R F 7$, along the longitudinal axis of the root that determines the priming of LR primordia $\operatorname{sites}^{23}$. In contrast, here we investigate the establishment of fully operating circadian rhythms, including all evening loop components, and coordination of circadian signals as new LR primordia develop in growing roots.

The circadian clock rephases during LR development. The observed circadian oscillations in the LR data set can be explained by two different hypotheses: (H1) circadian oscillations in the root bend are initiated at germination ${ }^{24}$ or, (H2) the root gravitropic stimulus or LR primordium initiation rephases the circadian clock in the root bends, possibly independent of other tissues. To distinguish between these hypotheses, we applied a gravitropic stimulus to a TOC1:LUC reporter line, and monitored luminescence from germination onwards in continuous light conditions (Fig. 3a-e). When no gravitropic stimulus was applied, root tip and stem initially oscillated in phase, but oscillations dampened after 2 days in all parts of the root (Fig. 3b), while oscillations still persist in leaf and stem tissue (Fig. 3c). After gravity stimulation, robust oscillations became visible in the root bend and root tip tissues and persist over the course of the experiment. Regions above the bend still dampened quickly, similar to the control data in absence of a gravitropic stimulus. When comparing the region at which root bending occurs to the robustly rhythmic root tip, it appears that the bending initially triggers an earlier phase (1-3h) and also a longer periodicity, suggesting that this region at the root bend is behaving differently to other parts of the root tissue and other parts of the plant (Fig. 3d,e). However, $48 \mathrm{~h}$ after gravity stimulation the phases of the root bend and root tip synchronize. This finding can be explained by recent data showing that the vascular tissues of cotyledons, where the circadian clock is distinct and more robust than in mesophyll tissues, can regulate gene expression as well as physiological responses in the neighbouring mesophyll tissue ${ }^{7}$. In the case of a root bend, $48 \mathrm{~h}$ after gravity stimulation the new

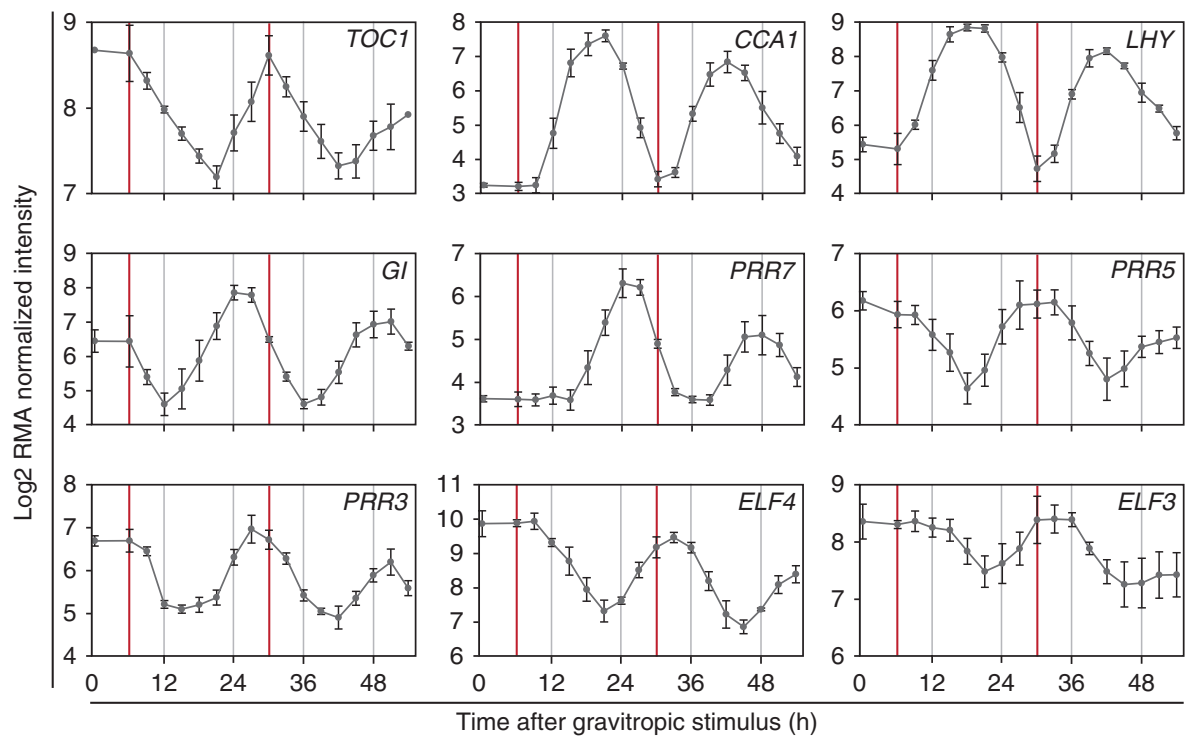

Figure 2 | Circadian oscillations in the lateral root time course. RNA expression patterns of the circadian clock genes CCA1, LHY, GI, TOC1, PRR7, PRR5, PRR3, ELF4 and ELF3, all of which oscillate in the lateral root data set. Expression intensities from the lateral root microarray data are on a log2 scale. Red vertical bars indicate peak times of TOC1 expression. $N=4$. All seedlings were grown in constant light conditions, without previous entrainment. The mean of the gene expression is given by the solid line and the error bars show the mean \pm 2 s.e. 
a

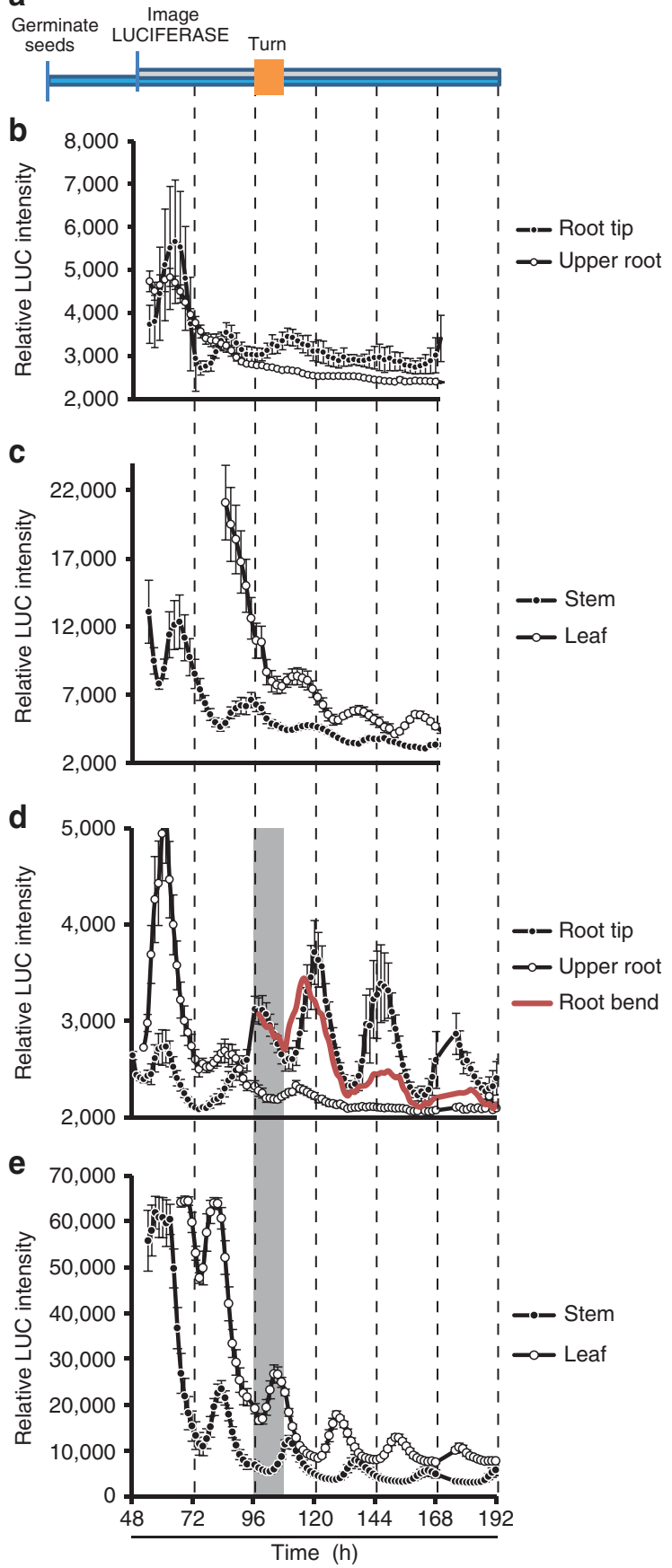

f

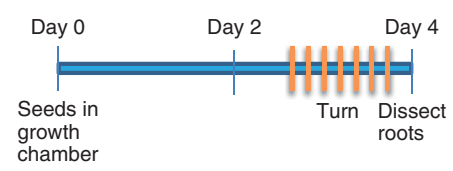

g

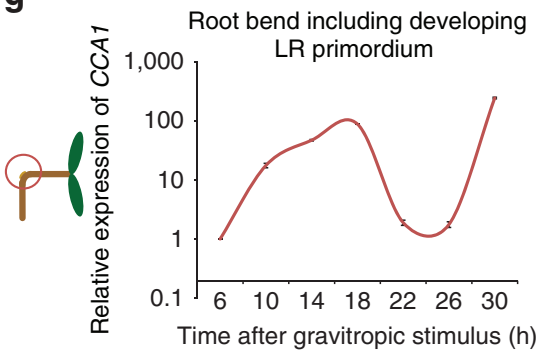

$h$

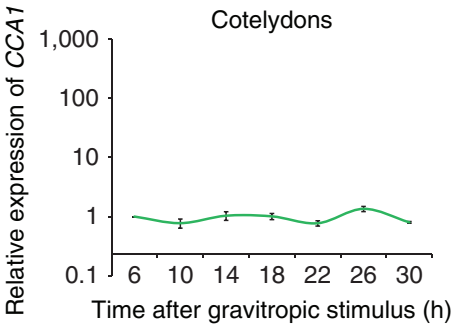

i

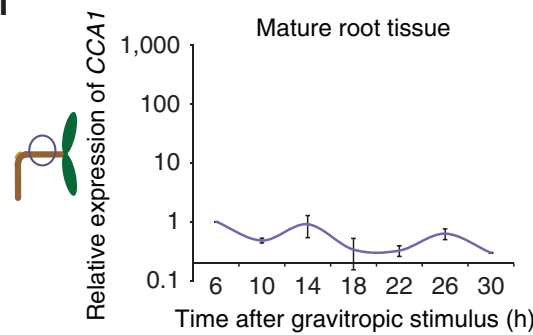

j

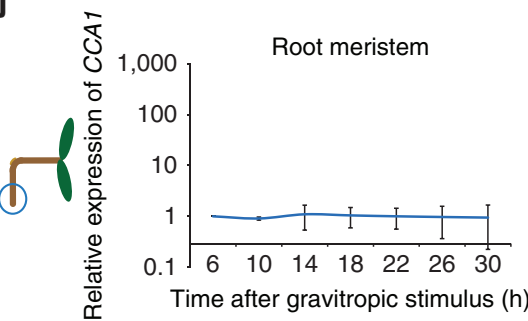

Figure 3 | Gravitropic stimulation regulates the circadian clock in the root bend. (a) Luminescence levels of TOC1:LUC plants were continuously monitored. TOC1:LUC seedlings were grown vertically and imaged after the seeds were placed on plates. (b,c) Luminescence of control seedlings without gravitropic stimulus was monitored in: root tip and upper root (b), stem and leaf (c). (d,e) In contrast to b,c after 4 days a $90^{\circ}$ gravitropic stimulus, as indicated by the grey section, was applied for $12 \mathrm{~h}$. After that period, the plate was rotated back to original start position. (b-e) Error bars indicate s.e.; $N=2$. (f) From seedlings that have been exposed to a gravitropic stimulus at different ages, various tissues (root bend ( $(\mathbf{g})$, cotyledons (h), upper root (i) and root meristem (j); indicated by circles) were dissected at the same time/age of the seedling (shown in $\mathbf{f}$ ). (g-j) RNA was prepared and RT-qPCR was used to measure the levels of CCA1 mRNA. Only the root bends show oscillation of CCA1 expression. Error bars indicate s.d.; $N=4$.

LR has emerged (Fig. 1b) and its vascular tissues have differentiated. We hypothesize that this maturation of the LR vascular tissues could explain the rephasing of the LR clock to the primary root clock. Irrespective, these data indicate that the LR circadian clock initially oscillates in a phase independent of the circadian clock of other root tissues.

To validate that the LR circadian clock does indeed oscillate in a phase independent of the circadian clock in other tissues, we conducted another experiment. Again, all seedlings were grown in continuous light conditions, without previous entrainment, consistent with the growth conditions in the previous experiments. To prove that both the morning and evening loops of the circadian clock rephase during LR development, we measured expression levels of the morning loop gene CCA1 in this experiment. In contrast to the microarray experiment (Fig. 1), all seedlings were dissected at the same age (same 
duration in the growth chamber), but gravity-stimulated at different ages post germination (Fig. 3f). From these plants, cotyledons, root bends, meristems and root sections above the bending region were dissected and CCA1 mRNA levels were measured by quantitative reverse transcription PCR (qRT-PCR; Fig. $3 g-j)$. If the gravistimulus did not change the circadian clock phase in the investigated tissue, one would expect a flat expression curve, as all samples would be harvested in the same phase of oscillation. However, if the gravistimulus leads to rephasing of the oscillator, one would observe CCA1 oscillations when plotting the data against 'time after gravitropic stimulus'. Our results reveal that $C C A 1$ transcript abundance starts to oscillate in the root bend itself (Fig. $3 \mathrm{~g}$ ), while in the other sampled tissues (Fig. $3 \mathrm{~h}-\mathrm{j}$ ) the mRNA profile of CCA1 was flat, confirming that the circadian clock rephases at the root bend after the gravitropic stimulus, independent of other plant tissues.

TOC1 is auxin-inducible during LR initiation. At the start of the LR time course, transcripts of the morning genes CCA1, LHY and PRR7 are low, whereas mRNAs of afternoon and evening expressed genes PRR5, PRR3, GI, TOC1, ELF3 and ELF4 are high (Fig. 2). The first significant changes in circadian clock transcript abundance are a rapid decline of TOC1 and GI versus a small increase in $L H Y$ mRNA abundance from $9 \mathrm{~h}$ pgi (Fig. 2). All other circadian clock genes start to oscillate later (Fig. 2), suggesting that TOC1, GI and/or LHY genes regulate rephasing of circadian clock oscillations in LRs. This pattern of transcript abundance is similar in dry seeds before imbibition ${ }^{24}$, which is believed to synchronize and set the phase of the clock in Arabidopsis $^{25}$. Since TOC1, GI and LHY are candidates for regulating circadian clock rephasing, and given that the plant hormone auxin triggers LR initiation, we investigated whether their expression is regulated by this hormone. Seedling roots were first treated with auxin (indole-3-acetic acid (IAA)) and then apical meristem and elongation zones (the latter containing the basal meristem from which new LR primordia originate) were microdissected, then the RNA isolated and profiled (as described for root bends). In the transcriptomic data generated using wild-type (WT) (Col-0) root material, TOC1 transcript abundance was rapidly induced nearly twofold $15 \mathrm{~min}$ after auxin treatment in the basal meristem where LRs are primed (Fig. 4a), while in the root meristem the induction is nearly absent (Supplementary Fig. 3a). To validate this observation, we transcript profiled equivalent auxin-treated root zones in the arf7arf19 mutant (Fig. 4a and Supplementary Fig. 3a). ARF7 and ARF19 are two (of a total of five) auxin response (transcription activating) factors in Arabidopsis that are required for LR initiation since arf7arf19 mutants do not form LRs ${ }^{12,15,16}$. As the auxin response pathway and LR development are disrupted in arf7arf19, we employed this double mutant background as a control for the auxin transcriptomic experiment. We observed that while ARF7 and ARF19 are not required for the initial rapid auxin-dependent upregulation of TOC1, they were essential for the maintenance of high TOC1 transcript levels (Fig. 4a). In contrast to TOC1 and $L H Y$, root transcript abundance was not significantly changed by auxin (Supplementary Fig. 3d,e). It has been demonstrated that high auxin concentrations can affect, but do not rephase the circadian $\operatorname{clock}^{26}$ and that pulses of CCA1 or LHY expression can shift the phase of circadian rhythms, but pulses of TOC1 expression do not ${ }^{27}$. In addition, TOC1 expression decreased after IAA treatment ${ }^{26}$. However, these observations were made in whole seedlings. As other major differences have been described for root and shoot circadian clock regulation $^{8}$, we cannot rule out that this mechanism might be different in roots or LR primordia in particular. The observed auxin induction of TOC1 gene expression cannot be seen in other auxin and LR time course data sets ${ }^{28-31}$. However, the time points sampled do not have the resolution to pick up the rapid induction of TOC1 (within $15 \mathrm{~min}$ ). In addition, whole seedlings or whole roots were sampled for those data sets, most likely resulting in a dilution of TOC1 induction, which is specific to the root basal meristem. Based on this data, we suggest that auxin controls the induction and maintenance of TOC1 expression, representing a novel role for auxin in LR circadian clock regulation.

The circadian clock is required for LR development. To determine whether a fully operating circadian clock is required for normal LR development, we compared LR densities in single mutants and overexpressing lines of the three core circadian clock genes CCA1 (cca1-1, CCA1-OX), LHY (lhy-21, LHY-OX) and TOC1 (toc1-1, TOC1-OX) (Fig. 4b, Supplementary Figs $4 a, c$ and $5 b-e)^{32-34}$. For phenotyping, all plants were grown in continuous light conditions, without previous entrainment, consistent with growth conditions from the previous experiments. The strongest reduction in emerged LR and LR primordium density was found in toc1-1, CCA1-OX and LHY-OX lines (between 30 and 60\%), whereas other lines displayed weaker LR developmental phenotypes (Fig. 4b, Supplementary Figs $4 \mathrm{~b}$ and 5b). While the absence of LR phenotypes in TOC1-OX and lhy-21 mutants seems puzzling on the first glance, a similar variety of phenotypic occurrence has been described for various other circadian clock-associated phenotypes. For example, CCA1$O X, L H Y-O X$, elf3, prr7 and prr9 exhibit long hypocotyl phenotypes, while the toc1-1 mutant does not ${ }^{3,35}$. Similar phenotypic discrepancies among circadian clock mutants have been shown in the control of flowering timing ${ }^{3}$. In addition, we attribute the phenotypic differences between these lines to toc1-1 being a semi-dominant missense allele (in its DNA-binding domain $)^{36}$ that disrupts the mutant toc $1-1$ protein binding to promoters such as CCA1 to repress their expression ${ }^{4}$, while CCA1 and LHY overexpression has been reported to be able to reset the phase of the circadian clock $^{27}$. To determine whether this phenotype arose as a result of a defect in LR initiation or development, we profiled the stages of LR primordia in 10-dayold seedlings in toc1-1, LHY-OX and CCA1-OX and compared them to their respective WTs. The proportion of toc1-1 LR primordia between stages I and IV was increased threefold compared with the WT control (C24, Fig. 4d), although the overall primordia density, including emerged LRs, was similar (Fig. 4c). LHY-OX lines also displayed a threefold increase in stage IV primordia (Supplementary Fig. 5c), but the overall primordia density was still lower than WT (Supplementary Fig. 5b). CCA1-OX lines did not display a strong LR primordium phenotype. These data suggest that disrupting the circadian clock leads to strong defects in LR development or emergence, but not initiation (Fig. 4b-d, Supplementary Figs 4 and 5). The transition from stage II to $\mathrm{V}$ is an important phase in LR development, since the Casparian strip, a tough lignified barrier that effectively ropes together endodermal cells overlying new LRs, needs to be broken to allow the new root organ to emerge ${ }^{37}$. Auxin is a key signal promoting LR emergence ${ }^{11,38,39}$. This hormone is transported from newly initiated LR primordia towards cells in overlying tissues, where it triggers the expression of genes including its own influx carrier, $L A X 3$, and the cell-wall remodelling enzyme polygalacturonase $^{11,14,40}$.

The LR circadian clock gates auxin levels and response. Given the LR development or emergence defects exhibited by mutant and overexpresser lines of core clock components, we examined 
a

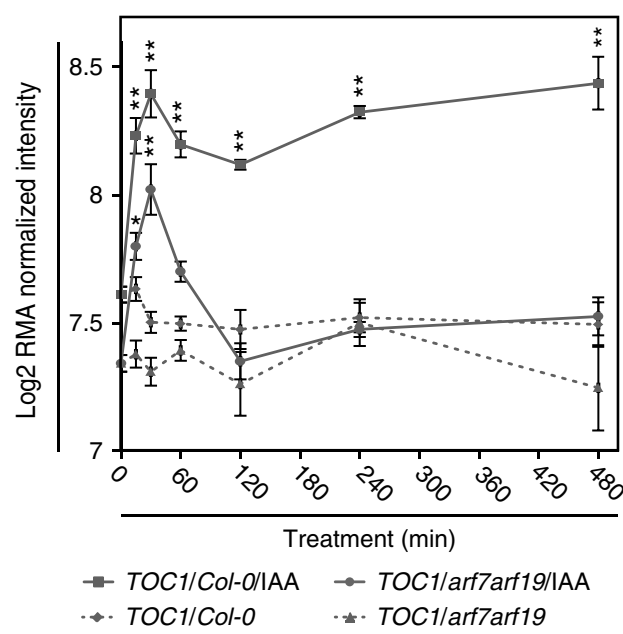

b

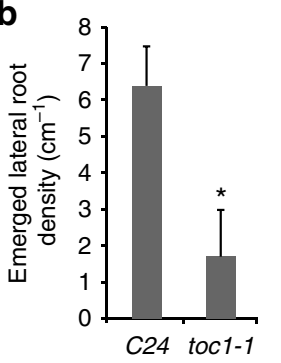

C
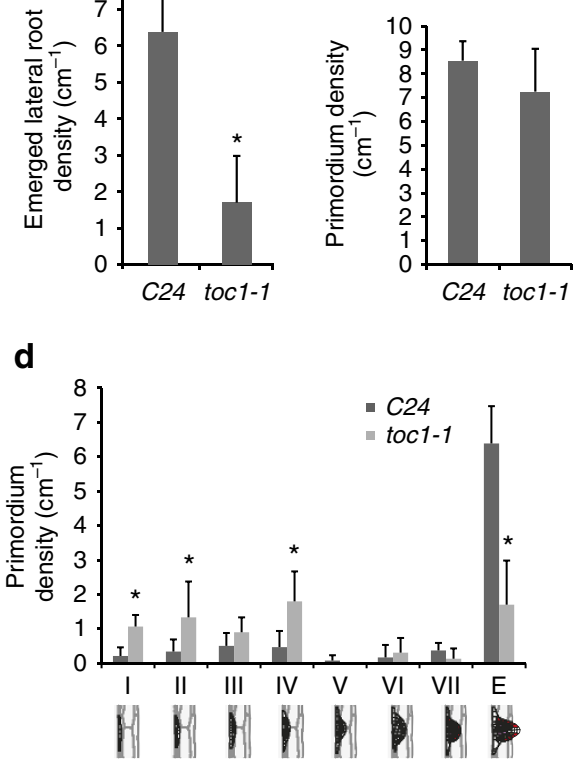

e

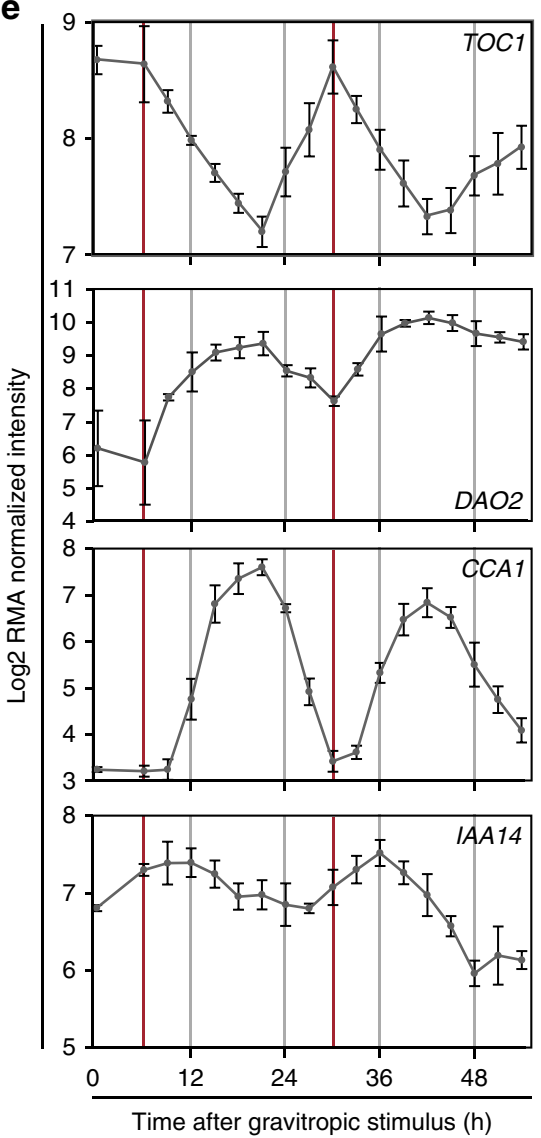

f

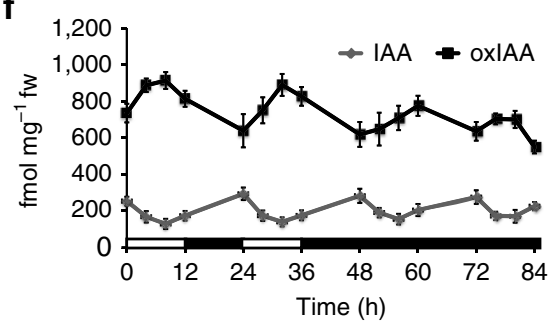

Figure 4 | Lateral root phenotypes of circadian clock mutants. (a) TOC1 gene expression in the basal meristem is induced in response to auxin treatment of whole seedlings in $\mathrm{Col}-\mathrm{O}$, as well as arf7arf19 mutant plants. Expression intensities are on a log2 scale and are based on transcriptomics data with an ATH1 chip (Affymetrix), three independent biological replicates each. Error bars indicate s.d.; asterisks indicate a significant difference $(P<0.05$,

Student's t-test) from wildtype. (b-d) All seedlings were grown in constant light conditions, without previous entrainment. $N=20$ for all experiments. Error bars indicate s.d.; asterisks indicate a significant difference from wildtype. (b) Emerged lateral root density of 10-day-old C24 and toc1-1 seedlings in $\mathrm{cm}^{-1}$ revealed a strong phenotype for toc1-1. (c,d) Ten-day-old seedlings were fixed and the primordium density of different developmental stages (stage I to emerged (E)) of wild-type (C24) and toc1-1 mutant seedlings in $\mathrm{cm}^{-1}$ was determined. (e) Comparison of oscillating gene expression patterns from the lateral root transcriptomic time course of the circadian clock genes CCA1 and TOC1 and auxin signalling genes IAA14 and AtDAO2, demonstrate gating of auxin signalling by the circadian clock during lateral root primordium development. Expression intensities are on a log2 scale. Red vertical bars indicate peak times of TOC1 expression. All seedlings were grown in constant light conditions, without previous entrainment. The mean of the gene expression is given by the solid line and the error bars show the mean \pm 2 s.e. $N=4$. (f) IAA and oxIAA accumulations exhibit circadian rhythms in roots under free-running conditions. Seedlings were germinated and entrained under $12 \mathrm{~h}$ light/dark cycle before transfer to constant darkness. Tissue was harvested starting at subjective dawn for 5 -day seedlings. The experiment was repeated three times. White bars, light period; dark bars, dark period. Error bars indicate s.d.; $N=3$.

whether the circadian clock may gate auxin signalling during LR development. We observed that 49 auxin-related genes display circadian oscillations during LR development under our experimental conditions ${ }^{21}$ (see Supplementary Data 3 for oscillating auxin-related genes). Among these auxin-related genes is IAA14 (Fig. 4e), which is a key regulator of $\operatorname{LR}_{\text {initiation }}{ }^{41}$ and emergence ${ }^{9}$. Significantly, expressing IAA14 under the regulation of the $35 S$ promoter blocks LR emergence ${ }^{42}$. Hence, replacing the native IAA14 gene's oscillatory pattern (Fig. 4e) with a constitutive mode of expression in 35S::IAA14 transgenic seedlings disrupts LR emergence ${ }^{42}$. IAA14 functions as a transcriptional repressor that interacts directly with ARF7 and ARF19 proteins. Hence, regulating IAA14 expression in a circadian manner (Fig. 4e) would serve to gate the activity of the IAA14/ARF7/ARF19 auxin response module and their downstream genes that include direct targets $L B D 16$ and LBD29 
(refs 43,44). Consistent with this model, LBD29, and to a lesser extent LBD16, are among the auxin-related genes whose mRNAs display circadian oscillations during LR development in our transcriptomic data set (Supplementary Fig. 1 and Supplementary Data 3).

AtDAO2 (AT1G14120) represents another strongly circadian oscillating auxin-related mRNA (Fig. 4e, Supplementary Data 3). AtDAO2 is orthologous to the rice DAO enzyme mediating degradation of the major plant auxin IAA to its biologically inactive form oxIAA ${ }^{45}$. Metabolic profiling of root tissues revealed that oxIAA levels display strong circadian oscillations under free-running conditions (after initial entrainment for 2 days), and which increases during the subjective day coincident with elevated AtDAO2 transcript abundance (Fig. 4f). In parallel, free IAA levels oscillate in anti-phase with AtDAO2 and oxIAA (Fig. 4e,f). The circadian patterns of AtDAO2 and IAA levels (Fig. 4e,f) would lead to the gating of auxin-inducible gene expression such as IAA14 during root development. Moreover, the turnover of the IAA14 protein is also auxin dependent ${ }^{46,47}$ creating further opportunities to gate auxin response activity during LR development. Indeed, slowing the auxin-dependent dynamics of IAA14 degradation was recently demonstrated to cause a quantitative reduction in LR emergence ${ }^{48}$.

AtDAO2 exhibits a circadian oscillation in transcript abundance similar to CCA1 but in anti-phase to TOC1 (Fig. 4e). AtDAO2 mRNA is initially induced one time step before CCA1, suggesting that its circadian expression pattern is not dependent on CCA1. The AtDAO2 promoter contains nine TOC1 (T1ME) binding motifs and TOC1 has recently been reported to be a transcriptional repressor ${ }^{4}$. In addition, AtDAO2 induction coincides with TOC1 transcript abundance dropping and vice versa, suggesting that $A t D A O 2$ is negatively regulated by TOC1 (Fig. 4e). TOC1 functioning to directly repress AtDAO2 expression could explain why the toc1-1 mutant exhibits such a severe LR phenotype (Fig. 4b-d, Supplementary Fig. 4) since expression of many other auxin regulated genes (in addition to AtDOA2) would be uncoupled from the circadian clock.

\section{Discussion}

We conclude that a fully operating circadian clock is necessary during LR emergence. Endo et al. ${ }^{7}$ recently proposed that circadian clocks of different tissues perform distinct functions. Consistent with this, AtDAO2 and 14 other auxin-related genes (including $L A X 3$ ) oscillating in our LR data set do not exhibit circadian changes in whole seedlings ${ }^{26}$ or cotyledons ${ }^{7}$ (Fig. 4e, Supplementary Fig. 1, Supplementary Data 3). Our results suggest that the circadian clock functions like a developmental 'metronome' during LR development, causing the expression of genes such as AtDOA2 and IAA14 to gate auxin responses in cells overlying new primordia, and thereby coordinate organ emergence. But why do the circadian clocks of cells in the vicinity of new LR primordia have to be rephased compared with other root tissues? One likely explanation is that cells within and surrounding new LR primordia need to independently regulate their hydraulic properties in a manner distinct to other root tissues to facilitate organ emergence. We recently demonstrated that auxin channelled via the LR primordium into overlying cells functions to repress the expression of genes encoding water channels termed aquaporins, creating complex spatio-temporal changes in hydraulic properties necessary for organ emergence ${ }^{14}$. In contrast, in other root tissues the circadian clock regulates aquaporin expression, resulting in diurnal oscillations in root water uptake ${ }^{49,50}$. Rephasing the circadian clock in cells within the new LRP and surrounding tissues would, in effect, transiently hydraulically isolate them from these other diurnal oscillations in root water uptake. Nevertheless, we observed that the circadian phases of the newly emerged lateral root primordium (LRP) and root tip eventually synchronize and hypothesize that the maturation of the vascular tissues in the new root organ could explain this rephasing (akin to the recently proposed role of the vasculature in cotyledons ${ }^{7}$ ).

\section{Methods}

Growth conditions and plant material. WT, mutants and reporter lines were grown vertically on sterile $12 \times 12 \mathrm{~cm}$ square Petri dishes on $1 / 2 \mathrm{MS}$ (Murashige and Skoog; pH 5.8) at $23^{\circ} \mathrm{C}$ under continuous light $\left(150 \mu \mathrm{mol} \mathrm{m}^{-2} \mathrm{~s}^{-1}\right)$. For RNA preparation, transcriptomic and phenotypic analysis, LR induction was performed on 3-day-old Col-0 seedlings grown vertically by rotating the plates by $90^{\circ}$. All mutant lines were obtained from the European Arabidopsis Stock Centre (NASC).

RNA extraction for LR transcriptomic data and qRT-PCR. Total RNA was extracted from roots using Qiagen RNeasy plant mini kit with on-column DNAse treatment following the manufacturer's recommended protocol (RNAse-free DNAse Set, Qiagen, Crawley, UK). RNA samples were quantified using a Nanodrop ND100 spectrophotometer (Nanodrop, Wilimington, USA).

Transcriptome analysis of LR development. Four biological replicates from separate pools of seeds ( Col-0) were stratified for 2 days at $4{ }^{\circ} \mathrm{C}$, before transfer to growth chambers. For every time point, root bends of $\sim 400$ seedlings were microdissected under a binocular microscope and frozen in liquid nitrogen immediately on harvesting as described in ref. 14. In addition to the root bends, a mature root segment located between the bend and the shoot was harvested at $9 \mathrm{~h}$ after an inductive gravitropic stimulation to serve as a reference of non-gravitropicstimulated root tissues devoid of developing LRP (time point 0 in the data set; for more details about timing of sampling see Supplementary Table 1). RNA labelling and hybridization to Affymetrix ATH1 arrays were performed by NASC.

Data were normalized as described in ref. 17. Error bars indicate s.d.; asterisks indicate a significant difference from WT. For timings of plant growth and root sampling see Supplementary Table 1, which applies to all four replicates. Microarray data are available in the ArrayExpress database (www.ebi.ac.uk/ arrayexpress) under accession number E-MTAB-2565.

Transcriptome analysis of WT and arf7arf19. Three biological replicates from separate pools of seeds (Col-0 and arf7arf19) were stratified for 2 days at $4{ }^{\circ} \mathrm{C}$, before transferring to growth chambers for 6 days. Sterile $9 \times 9 \mathrm{~cm}$ square sections of $100 \mu \mathrm{m}$ nylon mesh (Clarcor) were placed onto the media surface before sowing to facilitate root dissection and harvesting of cut sections. Plates were then transferred to tanks containing 31 of $0.5 \times$ MS media and allowed to acclimate for $24 \mathrm{~h}$ and then to tanks containing 31 of $0.5 \times$ MS media supplemented with either $30 \mu \mathrm{l} 100 \%$ ethanol or $30 \mu \mathrm{l}$ of $0.1 \mathrm{M}$ IAA in $100 \%$ ethanol $(1 \mu \mathrm{M}$ final IAA concentration) for $0,15,30,60,120,240$ or $480 \mathrm{~min}$.

For each biological replicate, plants were grown and $\sim 50$ roots were dissected into 2 sections; the meristem (from the root tip to the top of the LR cap, $\sim 350 \mu \mathrm{m}$ from the tip) and the basal meristem zone (from the top of the LR cap to the first visible root hair bulge, $\sim 850 \mu \mathrm{m}$ from the shootward boundary of zone 1 ). Dissected samples were immediately frozen in liquid nitrogen. RNA was extracted using the Qiagen MicroRNA Kit following the manufacturers recommended protocol (Qiagen) and quantified using a Nanodrop ND100 spectrophotometer. RNA labelling and hybridization to Affymetrix ATH1 arrays were performed by NASC.

Data were normalized as described in ref. 17. Transcriptomics data used in these experiments have been made available through ArrayExpress (www.ebi.ac.uk) with accession number E-MEXP-1354.

qRT-PCR. Poly(dT) complementary DNA (cDNA) was prepared from $2 \mu \mathrm{g}$ total RNA using the Transcriptor first strand cDNA synthesis kit (Roche). Quantitative PCR was performed using SYBR Green Sensimix (Quantace) on a Roche LightCycler 480 apparatus. PCR was carried out in 384-well optical reaction plates heated for $1 \mathrm{~min}$ to $95^{\circ} \mathrm{C}$, followed by 40 cycles of denaturation for $5 \mathrm{~s}$ at $95^{\circ} \mathrm{C}$, annealing for $8 \mathrm{~s}$ at $62^{\circ} \mathrm{C}$ and extension for $30 \mathrm{~s}$ at $72^{\circ} \mathrm{C}$. Target quantifications were performed with the specific primer pairs described in Supplementary Table 2. Expression levels were normalized to ACTIN. All qRT-PCR experiments were performed in quadruplicates and the values represent mean \pm s.e.m.

LR analysis and microscopy. Total number and stages of LRP were counted using primary roots cleared by immersion in $20 \%(\mathrm{v} / \mathrm{v})$ methanol $/ 4 \%(\mathrm{v} / \mathrm{v})$ hydrochloric acid at $57^{\circ} \mathrm{C}$ for $20 \mathrm{~min}$, followed by immersion in $7 \%(\mathrm{w} / \mathrm{v}) \mathrm{NaOH} / 60 \%$ (v/v) ethanol at room temperature for $15 \mathrm{~min}$. Roots were then rehydrated for $5 \mathrm{~min}$ each in $40 \%, 20 \%$ and $10 \%(\mathrm{v} / \mathrm{v})$ ethanol and mounted in $50 \%(\mathrm{v} / \mathrm{v})$ glycerol on glass microscope slides and were imaged using a Leica differential interference contrast optics microscope. 
Luciferase imaging. The TOC1:LUC reporter line was produced as part of the ROBuST project in a Col-0 background. Seedlings were grown on vertical $1 / 2 \mathrm{MS}$ plates at $23^{\circ} \mathrm{C}$ under continuous light. LR induction was performed on 3-day-old Col-0 seedlings grown vertically by rotating the plate by $90^{\circ}$. About $12 \mathrm{~h}$ later, the plate was rotated back to original start position. Luciferase imaging was done as described in ref. 51. Analysis of data was done using Imaris (Bitplane).

IAA/oxIAA measurements. A. thaliana Col-0 seeds were sown on $\frac{1}{4} \mathrm{MS}, 0.5 \%$ sucrose $\mathrm{pH} 5.7$ phytagar plates, and stratified at $4{ }^{\circ} \mathrm{C}$ for $48 \mathrm{~h}$. The seeds were transferred to a Conviron growth chamber, $120 \mu \mathrm{E}, 12 \mathrm{~h}$ day/night. Seedlings were harvested starting at day 5 . For free-running circadian experiments, samples were conducted as in the $12 \mathrm{~h}$ day/night experiments except that the growth chamber was shifted to $24 \mathrm{~h}$ darkness for the period described. Roots were collected, briefly patted dry, weighed in $1.5 \mathrm{ml}$ centrifuge tubes and frozen in liquid nitrogen and ground to powder. Samples were resuspended in $1 \mathrm{ml}$ of $50 \mathrm{mM}$ sodium-phosphate buffer, extracted for $20 \mathrm{~min}$ on a lab shaker at $4{ }^{\circ} \mathrm{C}$, and solid phase extraction was performed with $30 \mathrm{mg}$ Oasis HLB columns equilibrated with $50 \mathrm{mM}$ phosphate buffer, pH 3.0 as in ref. 52. Briefly, sample $\mathrm{pH}$ was adjusted to 3 with $\mathrm{HCl}$, applied to the HLB column, washed twice with $2 \mathrm{ml} 5 \%$ methanol, eluted with $2 \mathrm{ml} 80 \%$ methanol and dried under nitrogen. Standards added were $25 \mathrm{ng}$ 13C-IAA Cambridge Research Biochemicals) and indole-3-proprionic acid (Sigma). Calibration of indole3-proprionic acid and 13C-IAA was calculated with $25 \mathrm{ng}$ of oxindole-3-acetic acid (OlChemIm, Ltd., Olomouc, Czech Republic). Samples were resuspended in methanol and analysed by Liquid Chromatography-Multiple Reaction MonitoringMass Spectrometry (LC-MRM-MS) using an Agilent triple quad 6460. For each measurement, three samples were analysed. Results presented are means and s.d.

Differential expression. A gene was considered to be expressed if its expression was $>100$, and differentially expressed if a $t$-test between two time points was significant at a $q$ value of 0.05 after Benjamini-Hochberg false discovery rate correction $^{53}$. For most analyses, we further restricted this list to include genes that were only twofold induced or repressed ( $>8,000$ genes). More than $50 \%$ of probed genes $(>12,000)$ were significantly expressed in the main LR time course. Further analyses were performed using Excel 2010 (Microsoft Corporation, Redmond, USA).

Clustering. For each time course, we summarize the information contained in that profile by fitting a smoothing spline through the data. For details of smoothing splines, see ref. 54, p. 230. The basic idea is to fit a smooth curve through the data with a penalty on the roughness of the curve. The penalty is specified in the form of a multiplier of the integral of the squared second derivative of the curve in the objective function. We use the R function 'smooth spline' to fit the splines, and set the spar parameter to 0.4 (the multiplier described in the previous sentence is a monotone function of this parameter). We normalize each fitted spline to have mean 0 and variance 1 across the 18 time points of the experiment.

To cluster the normalized spline fits for differentially expressed genes in the LR time course data set, we use a method that combines hierarchical and $k$-means clustering. To cluster a set of several thousand genes, we proceed by breaking the data set-up into smaller blocks of 1,000 (plus 1 block containing $<1,000$ genes as the remainder). We cluster each block independently and then combine the centres of the different blocks to construct a set of centres on which we perform $k$-means clustering. The algorithm is as follows: calculate the spline fits for each gene and normalize to have mean 0 and variance 1; For a set of $N=1,000 B+R$ genes, where $B$ is the number of blocks of 1,000 and $R$ is the number $(<1,000)$ in the remainder, break the data set into $B$ blocks of 1,000 genes and 1 block of R genes; For each of the $(B+1)$ blocks, perform hierarchical clustering on the normalized splines using the Ward linkage and cutting the dendrogram at a height $h_{\max }$ to generate $(B+1)$ sets of centres, where each set will in general contain a different number of centres; Combine the sets of centres and cluster using hierarchical clustering, again using the Ward linkage and cutting the dendrogram at a height $2 \times h_{\max }$ to generate an overall set of centres; Use $k$-means clustering on the data set using the overall set of centres from the previous step as the initial centres in the algorithm.

This algorithm is computationally very fast, even for several thousand genes, taking only a few minutes to run. Initially, we applied the above algorithm (with $\left.h_{\max }=2\right)$ to the differentially expressed transcription factors (TFs) (1,304 genes) and to the other differentially expressed genes $(12,318$ genes) independently. This yielded a clustering with 45 centres for the TFs, and 174 centres for the non-TFs. A total of 219 clusters seemed very large, and possibly unnecessarily complex. We therefore decided to compare each of the non-TF centres to each of the $45 \mathrm{TF}$ centres. We used Euclidean distance between the vectors representing the centres as a measure of dissimilarity. We created a set of centres consisting of the $45 \mathrm{TF}$ centres together with the non-TF centres that were at least a distance of 2 away from all of the $45 \mathrm{TF}$ centres. We used the resulting set of 77 centres to perform $k$-means clustering of the entire set of differentially expressed genes. After obtaining the new centres following the $k$-means clustering, we summarized each cluster by taking the mean at each time point of each of its elements.

Estimating the period for oscillatory gene clusters. After using the spline clustering method outlined in the previous section, we identified 17 of the 77 clusters whose mean time profiles looked oscillatory. To estimate the period of these clusters, we proceeded as follows: for each oscillatory cluster, fit a smoothing spline through the data points with a spar parameter of 0.1 (which corresponds to a low level of smoothing, since we have already done the smoothing to obtain the initial centres for the $k$-means clustering); Use the predict.smooth.spline function in $\mathrm{R}$ to estimate the first derivative of the fitted curve at a grid of 1,000 points over the range of the length of the experiment ( $54 \mathrm{~h}$ in our case); If the derivative is exactly 0 at any of the time points on the grid, then use those time points to index the peaks and troughs. Otherwise use the average of neighbouring time points between which the sign of the first derivative changes as the indices of the turning points; For each set of peaks and troughs (turning points), calculate the time differences between neighbouring turning points, average these differences and double to obtain an estimate of the period for a particular oscillatory cluster; This analysis gives periods ranging between 19.4 and $35.9 \mathrm{~h}$, with six clusters showing periods within $1.5 \mathrm{~h}$ of $24 \mathrm{~h}$ (that is, between 22.5 and $25.5 \mathrm{~h}$ ).

\section{References}

1. Alabadi, D. et al. Reciprocal regulation between TOC1 and LHY/CCA1 within the Arabidopsis circadian clock. Science 293, 880-883 (2001).

2. Yakir, E. et al. Posttranslational regulation of CIRCADIAN CLOCK ASSOCIATED1 in the circadian oscillator of Arabidopsis. Plant Physiol. 150, 844-857 (2009).

3. Nagel, D. H. \& Kay, S. A. Complexity in the wiring and regulation of plant circadian networks. Curr. Biol. 22, R648-R657 (2012).

4. Gendron, J. M. et al. Arabidopsis circadian clock protein, TOC1, is a DNAbinding transcription factor. Proc. Natl Acad. Sci. USA 109, 3167-3172 (2012).

5. Huang, W. et al. Mapping the core of the Arabidopsis circadian clock defines the network structure of the oscillator. Science 336, 75-79 (2012).

6. Pokhilko, A. et al. The clock gene circuit in Arabidopsis includes a repressilator with additional feedback loops. Mol. Syst. Biol. 8, 574 (2012).

7. Endo, M., Shimizu, H., Nohales, M. A., Araki, T. \& Kay, S. A. Tissue-specific clocks in Arabidopsis show asymmetric coupling. Nature 515, 419-422 (2014)

8. James, A. B. et al. The circadian clock in Arabidopsis roots is a simplified slave version of the clock in shoots. Science 322, 1832-1835 (2008).

9. Lavenus, J. et al. Lateral root development in Arabidopsis: fifty shades of auxin Trends Plant. Sci. 18, 450-458 (2013).

10. Malamy, J. E. \& Benfey, P. N. Organization and cell differentiation in lateral roots of Arabidopsis thaliana. Development 124, 33-44 (1997).

11. Swarup, K. et al. The auxin influx carrier LAX3 promotes lateral root emergence. Nat. Cell Biol. 10, 946-954 (2008).

12. Peret, B. et al. AUX/LAX genes encode a family of auxin influx transporters that perform distinct functions during Arabidopsis development. Plant Cell 24 2874-2885 (2012).

13. Peret, B. et al. Sequential induction of auxin efflux and influx carriers regulates lateral root emergence. Mol. Syst. Biol. 9, 699 (2013).

14. Peret, B. et al. Auxin regulates aquaporin function to facilitate lateral root emergence. Nat. Cell Biol. 14, 991-998 (2012).

15. De Smet, I. et al. Bimodular auxin response controls organogenesis in Arabidopsis. Proc. Natl Acad. Sci. USA 107, 2705-2710 (2010).

16. Goh, T., Kasahara, H., Mimura, T., Kamiya, Y. \& Fukaki, H. Multiple AUX/ IAA-ARF modules regulate lateral root formation: the role of Arabidopsis SHY2/IAA3-mediated auxin signalling. Philos. Trans. R. Soc. Lond. B Biol. Sci. 367, 1461-1468 (2012).

17. De Rybel, B. et al. A novel aux/IAA28 signaling cascade activates GATA23dependent specification of lateral root founder cell identity. Curr. Biol. 20, 1697-1706 (2010).

18. Goh, T., Joi, S., Mimura, T. \& Fukaki, H. The establishment of asymmetry in Arabidopsis lateral root founder cells is regulated by LBD16/ASL18 and related LBD/ASL proteins. Development 139, 883-893 (2012).

19. Himanen, K. et al. Auxin-mediated cell cycle activation during early lateral root initiation. Plant Cell 14, 2339-2351 (2002).

20. Stacklies, W., Redestig, H., Scholz, M., Walther, D. \& Selbig, J. pcaMethods-a bioconductor package providing PCA methods for incomplete data. Bioinformatics 23, 1164-1167 (2007).

21. Hughes, M. E., Hogenesch, J. B. \& Kornacker, K. JTK_CYCLE: an efficient nonparametric algorithm for detecting rhythmic components in genome-scale data sets. J. Biol. Rhythms 25, 372-380 (2010).

22. Fukuda, H., Ukai, K. \& Oyama, T. Self-arrangement of cellular circadian rhythms through phase-resetting in plant roots. Phys. Rev. E Stat. Nonlin. Soft Matter Phys. 86, 041917 (2012).

23. Moreno-Risueno, M. A. et al. Oscillating gene expression determines competence for periodic Arabidopsis root branching. Science 329, 1306-1311 (2010).

24. Penfield, S. \& Hall, A. A role for multiple circadian clock genes in the response to signals that break seed dormancy in Arabidopsis. Plant Cell 21, 1722-1732 (2009).

25. Zhong, H. H., Painter, J. E., Salome, P. A., Straume, M. \& McClung, C. R. Imbibition, but not release from stratification, sets the circadian clock in Arabidopsis seedlings. Plant Cell 10, 2005-2017 (1998). 
26. Covington, M. F. \& Harmer, S. L. The circadian clock regulates auxin signaling and responses in Arabidopsis. PLoS Biol. 5, e222 (2007).

27. Knowles, S. M., Lu, S. X. \& Tobin, E. M. Testing time: can ethanol-induced pulses of proposed oscillator components phase shift rhythms in Arabidopsis? J. Biol. Rhythms 23, 463-471 (2008).

28. Vanneste, S. et al. Cell cycle progression in the pericycle is not sufficient for SOLITARY ROOT/IAA14-mediated lateral root initiation in Arabidopsis thaliana. Plant Cell 17, 3035-3050 (2005).

29. Okushima, Y. et al. Functional genomic analysis of the AUXIN RESPONSE FACTOR gene family members in Arabidopsis thaliana: unique and overlapping functions of ARF7 and ARF19. Plant Cell 17, 444-463 (2005).

30. Overvoorde, P. J. et al. Functional genomic analysis of the AUXIN/INDOLE-3ACETIC ACID gene family members in Arabidopsis thaliana. Plant Cell 17, 3282-3300 (2005).

31. Lewis, D. R. et al. A kinetic analysis of the auxin transcriptome reveals cell wall remodeling proteins that modulate lateral root development in Arabidopsis. Plant Cell 25, 3329-3346 (2013).

32. Millar, A. J., Carre, I. A., Strayer, C. A., Chua, N. H. \& Kay, S. A. Circadian clock mutants in Arabidopsis identified by luciferase imaging. Science 267, 1161-1163 (1995)

33. Green, R. M. \& Tobin, E. M. Loss of the circadian clock-associated protein 1 in Arabidopsis results in altered clock-regulated gene expression. Proc. Natl Acad. Sci. USA 96, 4176-4179 (1999).

34. Schaffer, R. et al. The late elongated hypocotyl mutation of Arabidopsis disrupts circadian rhythms and the photoperiodic control of flowering. Cell 93, 1219-1229 (1998)

35. Mas, P. Circadian clock signaling in Arabidopsis thaliana: from gene expression to physiology and development. Int. J. Dev. Biol. 49, 491-500 (2005).

36. Strayer, C. et al. Cloning of the Arabidopsis clock gene TOC1, an autoregulatory response regulator homolog. Science 289, 768-771 (2000).

37. Vermeer, J. E. et al. A spatial accommodation by neighboring cells is required for organ initiation in Arabidopsis. Science 343, 178-183 (2014).

38. Benkova, E. et al. Local, efflux-dependent auxin gradients as a common module for plant organ formation. Cell 115, 591-602 (2003).

39. Lucas, M. et al. Lateral root morphogenesis is dependent on the mechanical properties of the overlaying tissues. Proc. Natl Acad. Sci. USA 110, 5229-5234 (2013).

40. Kumpf, R. P. et al. Floral organ abscission peptide IDA and its HAE/HSL2 receptors control cell separation during lateral root emergence. Proc. Natl Acad. Sci. USA 110, 5235-5240 (2013).

41. Fukaki, H., Nakao, Y., Okushima, Y., Theologis, A. \& Tasaka, M. Tissue-specific expression of stabilized SOLITARY-ROOT/IAA14 alters lateral root development in Arabidopsis. Plant J. 44, 382-395 (2005).

42. Fukaki, H., Tameda, S., Masuda, H. \& Tasaka, M. Lateral root formation is blocked by a gain-of-function mutation in the SOLITARY-ROOT/IAA14 gene of Arabidopsis. Plant J. 29, 153-168 (2002).

43. Okushima, Y., Fukaki, H., Onoda, M., Theologis, A. \& Tasaka, M. ARF7 and ARF19 regulate lateral root formation via direct activation of LBD/ASL genes in Arabidopsis. Plant Cell 19, 118-130 (2007).

44. Lavenus, J. et al. Inference of the Arabidopsis lateral root gene regulatory network suggests a bifurcation mechanism defines primordia flanking and central zones. Plant Cell 27, 1368-1388 (2015).

45. Zhao, Z. et al. A role for a dioxygenase in auxin metabolism and reproductive development in rice. Dev. Cell 27, 113-122 (2013).

46. Dharmasiri, N., Dharmasiri, S. \& Estelle, M. The F-box protein TIR1 is an auxin receptor. Nature 435, 441-445 (2005).

47. Kepinski, S. \& Leyser, O. The Arabidopsis F-box protein TIR1 is an auxin receptor. Nature 435, 446-451 (2005).

48. Guseman, J. M. et al. Auxin-induced degradation dynamics set the pace for lateral root development. Development 142, 905-909 (2015).

49. Caldeira, C. F., Jeanguenin, L., Chaumont, F. \& Tardieu, F. Circadian rhythms of hydraulic conductance and growth are enhanced by drought and improve plant performance. Nat. Commun. 5, 5365 (2014)

50. Takase, T. et al. The circadian clock modulates water dynamics and aquaporin expression in Arabidopsis roots. Plant Cell Physiol. 52, 373-383 (2011).
51. Gould, P. D. et al. The molecular basis of temperature compensation in the Arabidopsis circadian clock. Plant Cell 18, 1177-1187 (2006).

52. Novak, O. et al. Tissue-specific profiling of the Arabidopsis thaliana auxin metabolome. Plant J. 72, 523-536 (2012).

53. Smyth, G. K. in Bioinformatics and Computational Biology Solutions Using $\mathrm{R}$ and Bioconductor, (eds Gentleman R., Carey V., Dudoit S., Irizarry R. and Huber W.) 397-420. (Springer, 2005).

54. Venables, W. N. \& Ripley, B. D. Modern Applied Statistics with S (Springer, 2002).

\section{Acknowledgements}

We thank Andrew J. Millar, Philip N. Benfey, Guy Wachsman and Jaimie M. Van Norman for useful comments and suggestions and Amber Chase for growing plants for oxIAA profiling. U.V., K.K., M.H.W., K.S., T.J.H., D.M.W., B.P., T.C.H., M.L. and M.J.B. acknowledge the support of the Biotechnology and Biological Sciences Research Council (BBSRC) and Engineering and Physical Sciences Research Council (EPSRC) funding to the Centre for Plant Integrative Biology (CPIB) BB/D019613/1; U.V, K.S. and M.J.B. acknowledge funding from BBSRC grant BB/H020314/1; K.K., M.H.W. and K.S. acknowledge the support of the European Research Community Advanced Investigator Funding to M.J.B (FUTUREROOTS); D.M.W. and M.J.B. acknowledge BBSRC funding from grant BB/J009717/1 and U.V. and D.M.W. from the BBSRC Professorial Research Fellowship funding to M.J.B. grant BB/G023972/1; JSPS Fellowship to T.G., H.F. and M.J.B.; BBSRC funding for F.C.R and A.A.R.W. from grant BB/D017904/1; and Royal Society-Wolfson Merit Award to M.J.B. Work at the University of Liverpool by P.D.G and A.J.H was supported by the BBSRC/EPSRC-funded ROBuST SABR project BB/ F005318/1 and BBSRC grant BB/K018078/1. M.L. and L.L. were supported by the Région Languedoc Roussillon ('Chercheur d'Avenir' grant to LL) and the Agropolis Fondation Rhizopolis project. B.P. acknowledges the support of an Intra-European Fellowship for Career Development under the seventh framework of the European Commission (IEF2008-220506), an EMBO Long-Term Fellowship, a European Reintegration Grant under the seventh framework of the European Commission (ERG-2010-276662). K.L. acknowledges the Swedish Governmental Agency for Innovation Systems (VINNOVA) and the Swedish Research Council (VR). W.A.P. and A.S.M. acknowledge funding from the Maryland Agricultural Experiment Station and DOE Physical Biosciences Basic Energy Sciences (DE-FG02-13ER16405).

\section{Author contributions}

U.V., M.H.W., K.K., P.D.G., W.A.P., M.L., K.S., I.C., T.J.H., D.M.W., B.P. T.G. and A.S.M. planned and performed the experiments; U.V., M.H.W., K.K., P.D.G., F.C.R. and D.M.W. analysed the data; U.V., M.H.W., K.K. and D.M.W. wrote the manuscript. H.F., T.C.H., L.L., K.J.H., K.L., A.J.H., A.S.M., A.A.R.W. and M.J.B. planned the research and wrote the manuscript. All the authors read the manuscript before submission.

\section{Additional information}

Accession codes. The microarray data is publicly available at ArrayExpress (www.ebi.ac.uk/arrayexpress/) under the accession numbers E-MTAB-2565 (for the lateral root time course) and E-MTAB-1354 (for the auxin time course in Fig. 4c and Supplementary Fig. 3).

Supplementary Information accompanies this paper at http://www.nature.com/ naturecommunications

Competing financial interests: The authors declare no competing financial interests.

Reprints and permission information is available online at http://npg.nature.com/ reprintsandpermissions/

How to cite this article: Voß, U. et al. The circadian clock rephases during lateral root organ initiation in Arabidopsis thaliana. Nat. Commun. 6:7641 doi: 10.1038/ncomms8641 (2015).

This work is licensed under a Creative Commons Attribution 4.0 International License. The images or other third party material in this article are included in the article's Creative Commons license, unless indicated otherwise in the credit line; if the material is not included under the Creative Commons license, users will need to obtain permission from the license holder to reproduce the material To view a copy of this license, visit http://creativecommons.org/licenses/by/4.0/ 